\title{
The $g$-extra edge-connectivity of balanced hypercubes
}

\author{
Yulong Weia ${ }^{a *}$ Rong-hua Li ${ }^{b}$ Weihua Yang ${ }^{\mathrm{a}}$ \\ ${ }^{a}$ Department of Mathematics, Taiyuan University of Technology, Taiyuan, 030024, China \\ ${ }^{\mathrm{b}}$ School of Computer Science \& Technology, Beijing Institute of Technology, Beijing, 100081, China
}

\begin{abstract}
The $g$-extra edge-connectivity is an important measure for the reliability of interconnection networks. Recently, Yang et al. [Appl. Math. Comput. 320 (2018) 464-473] determined the 3-extra edge-connectivity of balanced hypercubes $B H_{n}$ and conjectured that the $g$-extra edge-connectivity of $B H_{n}$ is $\lambda_{g}\left(B H_{n}\right)=2(g+1) n-4 g+4$ for $2 \leq g \leq 2 n-1$. In this paper, we confirm their conjecture for $n \geq 6-\frac{12}{g+1}$ and $2 \leq g \leq 8$, and disprove their conjecture for $n \geq \frac{3 e_{g}\left(B H_{n}\right)}{g+1}$ and $9 \leq g \leq 2 n-1$, where $e_{g}\left(B H_{n}\right)=\max \left\{\left|E\left(B H_{n}[U]\right)\right|\left|U \subseteq V\left(B H_{n}\right),\right| U \mid=g+1\right\}$.
\end{abstract}

Keywords balanced hypercube, $g$-extra edge-connectivity, reliability evaluation

\section{Introduction}

The topology of interconnection networks can be modeled by a graph $G=(V, E)$ in which a vertex represents a processor and an edge represents a communication link between processors. We refer readers to $[1,10,11]$ for terminology and notation unless stated otherwise. Once a network is running, some processors or links might be faulty. An interconnection network without faults is impossible. So the reliability evaluation of interconnection networks is significant.

${ }^{*}$ Corresponding author. E-mail address: weiyulong@tyut.edu.cn (Y. Wei). 
The traditional edge-connectivity $\lambda(G)$ is a measurement for the reliability of interconnection networks. However, in real situation, it is a small probability event that all links incident with the same processor fail simultaneously. To overcome this shortcoming, Esfahanian and Hakimi [2] proposed restricted edge-connectivity. Given a graph $G$, an edge-cut $S \subseteq E(G)$ is called a restricted edge-cut if there are no isolated vertices in $G-S$. The restricted edge-connectivity $\lambda^{\prime}(G)$ is the minimum cardinality of all restricted edge-cuts. Inspired by the restricted edge-connectivity, Fàbrega and Foil [3] proposed the $g$-extra edge-connectivity of a graph. We restate this concept as follows.

- Given a graph $G$, an edge-cut $F$ is called a $g$-extra edge-cut if every component of $G-F$ has at least $g+1$ vertices. The $g$-extra edge-connectivity of $G$, denoted by $\lambda_{g}(G)$, is the minimum cardinality of all $g$-extra edge-cuts, if exist.

A connected graph $G$ is called $\lambda_{g}$-connected if $\lambda_{g}(G)$ exists.

In recent years, the $g$-extra edge-connectivity of a graph has received much attention [5-7, 12-16, 19]. For example, Montejano and Sau [7] proved that given a connected graph $G$ and a positive integer $g$, determining $\lambda_{g}(G)$ or giving a correct report that $G$ is not $\lambda_{g}$-connected is NP-hard. Yang [13] determined that the 1-extra edge-connectivity of balanced hypercubes $B H_{n}$ is $\lambda_{1}\left(B H_{n}\right)=4 n-2$ for $n \geq 2$. L $\ddot{u}$ [6] showed that $\lambda_{2}\left(B H_{n}\right)=6 n-4$ for $n \geq 2$. Li et al. [5] and Yang et al. [12] independently proved that $\lambda_{3}\left(B H_{n}\right)=8 n-8$ for $n \geq 2$. In addition, Yang et al. [12] proposed a conjecture about the $g$-extra edge-connectivity of $\mathrm{BH}_{n}$ as follows.

Conjecture 1.1 Let $B H_{n}$ be an n-dimensional balanced hypercube. Then $\lambda_{g}\left(B H_{n}\right)=$ $2(g+1) n-4 g+4$ for $2 \leq g \leq 2 n-1$.

Let $e_{g}(G)=\max \{|E(G[U])||U \subseteq V(G)| U \mid,=g+1\}$, where $G[U]$ is the subgraph of $G$ induced by $U$. In this paper, we confirm their conjecture for $n \geq 6-\frac{12}{g+1}$ and $2 \leq g \leq 8$, and disprove their conjecture for $n \geq \frac{3 e_{g}\left(B H_{n}\right)}{g+1}$ and $9 \leq g \leq 2 n-1$. 


\section{Balanced hypercubes}

In 1997, Wu and Huang proposed balanced hypercubes $B H_{n}$.

Definition 2.1 ([9]) An n-dimensional balanced hypercube $B H_{n}=\left(V\left(B H_{n}\right), E\left(B H_{n}\right)\right)$ has vertex set $V\left(B H_{n}\right)=\left\{\left(a_{0}, a_{1}, \ldots, a_{i}, \ldots, a_{n-1}\right) \mid a_{i} \in\{0,1,2,3\}, 0 \leq i \leq n-1\right\}$. Each vertex $\left(a_{0}, a_{1}, \ldots, a_{i-1}, a_{i}, a_{i+1}, \ldots, a_{n-1}\right)$ of $B H_{n}$ has $2 n$ neighbors:

(1) $\left(\left(a_{0} \pm 1\right) \bmod 4, a_{1}, \ldots, a_{i-1}, a_{i}, a_{i+1}, \ldots, a_{n-1}\right)$,

(2) $\left(\left(a_{0} \pm 1\right) \bmod 4, a_{1}, \ldots, a_{i-1},\left(a_{i}+(-1)^{a_{0}}\right) \bmod 4, a_{i+1}, \ldots, a_{n-1}\right)$.

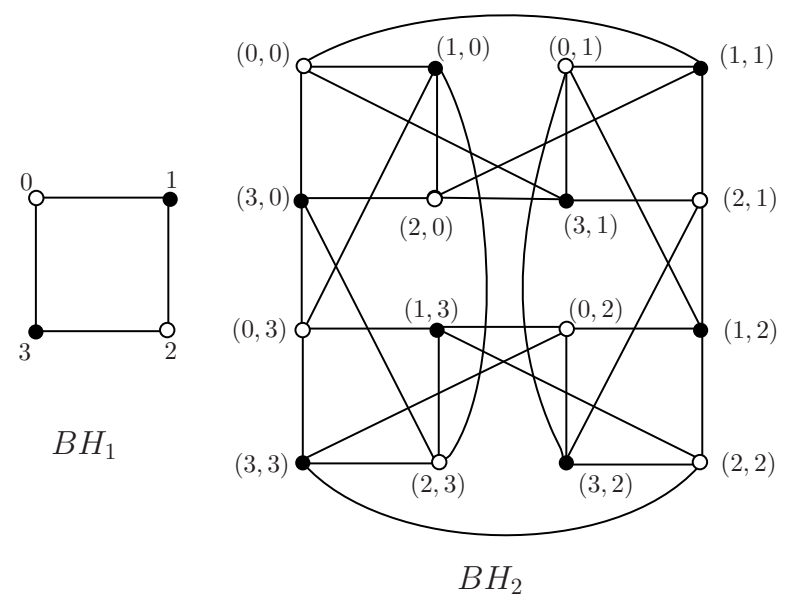

Figure 1: Illustration of $B H_{1}$ and $B H_{2}$.

Figure 1 depicts $B H_{1}$ and $B H_{2}$. Clearly, $B H_{n}$ is a $2 n$-regular graph. For a graph $G$ and a vertex $v \in V(G)$, the set of neighbors of $v$ in $G$ is denoted by $N_{G}(v)$. Some useful properties of $\mathrm{BH}_{n}$ are listed below.

Lemma 2.2 ([9]) The balanced hypercube $B H_{n}$ is bipartite.

Lemma 2.3 ([13]) Let $u$ be an arbitrary vertex of $B H_{n}$ for $n \geq 1$. Then, for an arbitrary vertex $v$ of $B H_{n}$, either $\left|N_{B H_{n}}(u) \cap N_{B H_{n}}(v)\right|=0,\left|N_{B H_{n}}(u) \cap N_{B H_{n}}(v)\right|=2$, or $\left|N_{B H_{n}}(u) \cap N_{B H_{n}}(v)\right|=2 n$. Furthermore, there is exactly one vertex $w$ such that $\left|N_{B H_{n}}(u) \cap N_{B H_{n}}(w)\right|=2 n$. 
According to Lemma 2.3, we call the vertex $w$ the equivalent vertex of $u$, denoted by $u^{\prime}$, if $w$ satisfies that $\left|N_{B H_{n}}(u) \cap N_{B H_{n}}(w)\right|=2 n$ in $B H_{n}$, and $u$ and $u^{\prime}$ are said to be a pair of equivalent vertices.

The following two lemmas are important observations about the structure of $B H_{n}$.

Lemma 2.4 The balanced hypercube $B H_{n}$ is $K_{3,3}$ free.

Proof. Assume to the contrary that there exists a subgraph $H_{1}$ of $B H_{n}$ which is isomorphic to $K_{3,3}$. By Lemma 2.2, suppose the bipartite graph $H_{1}=\left(X_{1}, Y_{1}\right)$, where $X_{1}=\left\{u_{1}, u_{2}, u_{3}\right\}$ and $Y_{1}$ are two parts of $H_{1}$. Since $N_{H_{1}}\left(u_{1}\right) \cap N_{H_{1}}\left(u_{2}\right)=Y_{1}, \mid N_{B H_{n}}\left(u_{1}\right) \cap$ $N_{B H_{n}}\left(u_{2}\right)|\geq| N_{H_{1}}\left(u_{1}\right) \cap N_{H_{1}}\left(u_{2}\right) \mid=3>2$. Thus, by Lemma 2.3, the vertex $u_{2}$ is the unique equivalent vertex of $u_{1}$. Similar to the above deduction, we see that the vertex $u_{3}$ is also the unique equivalent vertex of $u_{1}$, which contradicts $u_{2} \neq u_{3}$.

This completes the proof of Lemma 2.4.

Let $\mathcal{F}_{g}$ be a collection of induced subgraphs of $B H_{n}$ with $g+1$ vertices and $e_{g}\left(B H_{n}\right)$ edges for $g \geq 2$. By Lemma 2.2, $H$ is bipartite for any graph $H \in \mathcal{F}_{g}$.

Lemma 2.5 The vertex set $X$ (or $Y$ ) must consist of several pairs of equivalent vertices besides at most one vertex for some $H=(X, Y) \in \mathcal{F}_{g}$.

Proof. If $|X|=1$, then this lemma holds obviously. Now we consider the case of $|X| \geq 2$. Assume to the contrary that there exist two vertices $u, v \in X$ such that their equivalent vertices are not in $X$ for any graph $H=(X, Y) \in \mathcal{F}_{g}$. Without loss of generality, assume that $\left|N_{H}(u)\right| \geq\left|N_{H}(v)\right|$. We replace $v$ with $u^{\prime}$ and obtain an induced subgraph $H^{\prime}=\left(X^{\prime}, Y^{\prime}\right)$ of $B H_{n}$. If $\left|N_{H}(u)\right|>\left|N_{H}(v)\right|$, then $|V(H)|=\left|V\left(H^{\prime}\right)\right|$ and $|E(H)|<\left|E\left(H^{\prime}\right)\right|$, which contradicts the selection of $H$. If $\left|N_{H}(u)\right|=\left|N_{H}(v)\right|$ , then $H^{\prime} \in \mathcal{F}_{g}$. If $X^{\prime}$ contains a pair of vertices like $u, v \in X$, then this operation continues until we obtain a graph $H^{*}=\left(X^{*}, Y^{*}\right)$ satisfying that $X^{*}$ consists of several 
pairs of equivalent vertices besides at most one vertex. Note that $H^{*}=\left(X^{*}, Y^{*}\right) \in \mathcal{F}_{g}$, a contradiction.

By the similar arguments as above, we see that the vertex set $Y$ also consists of several pairs of equivalent vertices besides at most one vertex.

The lexicographic product $G \circ H$ of graphs $G$ and $H$ is defined as the graph with vertex set $V(G) \times V(H)$ and $\left(u_{1}, v_{1}\right)\left(u_{2}, v_{2}\right) \in E(G \circ H)$ if and only if $u_{1} u_{2} \in E(G)$, or $u_{1}=u_{2}$ and $v_{1} v_{2} \in E(H)$. Zhou et al. [17] proved that $B H_{n}$ is a lexicographic product of a Cayley graph $X_{n}$ and an empty graph with two vertices. In addition, Zhou et al. [18] showed that $B H_{n}$ is edge-transitive. Their results are presented as follows.

Lemma 2.6 ([17]) For each $n \geq 1, B H_{n} \cong X_{n} \circ 2 K_{1}$.

Lemma 2.7 ([17, Page 151]) For $n \geq 3$, the girth of $X_{n}$ is 6 .

Lemma 2.8 ([18]) The balanced hypercube is edge-transitive.

\section{Main Results}

In this section, we will discuss the $g$-extra edge-connectivity of the balanced hypercube $B H_{n}$ for $2 \leq g \leq 2 n-1$.

Let $G=(V, E)$ be a graph. For a nonempty proper subset $U \subseteq V$, the set of edges with one end in $U$ and the other end in $\bar{U}=V \backslash U$ is denoted by $[U, \bar{U}]$ and $\partial(U)=|[U, \bar{U}]|$. The $g$-th isoperimetric edge-connectivity $\gamma_{g}(G)$ of a graph $G$ was proposed by Hamidoune et al. [4]. We restate the definition of $\gamma_{g}(G)$, that is $\gamma_{g}(G)=\min \{\partial(U)|U \subseteq V| U \mid, \geq$ $g+1,|\bar{U}| \geq g+1\}$. Wang and Li $[8]$ gave a sufficient condition to ensure a regular edge-transitive graph such that $\lambda_{g}(G)=\gamma_{g}(G)$.

Lemma 3.1 ([8]) Let $G$ be a $k$-regular edge-transitive graph of order $n$ with $k \geq 2$, and let $g+1$ be a positive integer. If $n \geq 3(g+1)$, then $G$ is $\lambda_{g}$-connected, and $\lambda_{g}(G)=\gamma_{g}(G)$. 
A graph $G$ satisfying that $\gamma_{j}(G)=\beta_{j}(G)(j=0,1, \ldots, g)$ is called $\gamma_{g}$-optimal, where $\beta_{g}(G)=\min \{\partial(U)|U \subseteq V| U \mid,=g+1\}$. Zhang [16] gave a sufficient condition for a regular edge-transitive graph to be $\gamma_{g}$-optimal.

Lemma 3.2 ([16]) Let $g+1$ be a positive integer, and $G$ a connected $k$-regular edgetransitive graph with $k \geq \frac{6 e_{g}(G)}{g+1}$. Then $G$ is $\gamma_{g}$-optimal.

The following lemma gives a lower bound of $e_{g}\left(B H_{n}\right)$ for $2 \leq g \leq 2 n-1$.

Lemma 3.3 The balanced hypercube $B H_{n}$ satisfies that $e_{g}\left(B H_{n}\right) \geq 2 g-2$ for $2 \leq g \leq$ $2 n-1$.

Proof. Suppose that $u=(0,0, \ldots, 0), u^{\prime}=(2,0, \ldots, 0), u_{1}=(1,0, \ldots, 0), u_{2}=$ $(3,0, \ldots, 0), u_{2 i-1}=(1, \overbrace{0, \ldots, 0}^{i-2}, 1,0, \ldots, 0)$ and $u_{2 i}=(3, \overbrace{0, \ldots, 0}^{i-2}, 1,0, \ldots, 0)$ for $2 \leq$ $i \leq g-1$ are some vertices of $B H_{n}$. Let $A_{g}=\left\{u, u^{\prime}\right\} \cup\left\{u_{i} \mid 1 \leq i \leq g-1\right\}$. By Definition 2.1, we know that the induced subgraph $B H_{n}\left[A_{g}\right]$ is isomorphic to $K_{2, g-1}$ (see Figure 2). Therefore, $e_{g}\left(B H_{n}\right) \geq 2 g-2$ for $2 \leq g \leq 2 n-1$.

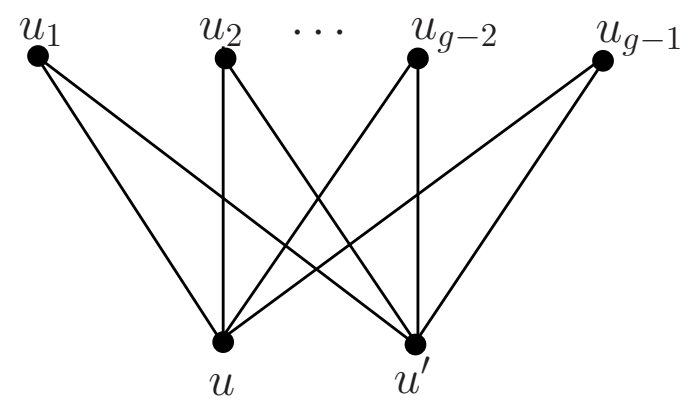

Figure 2: Illustration of $B H_{n}\left[A_{g}\right]$.

Now, we determine $e_{g}\left(B H_{n}\right)$ for $2 \leq g \leq 8$.

Lemma 3.4 The balanced hypercube $B H_{n}$ satisfies that $e_{g}\left(B H_{n}\right)=2 g-2$ for $2 \leq g \leq 8$. 
Proof. By Lemma 3.3, we only need to prove that $e_{g}\left(B H_{n}\right) \leq 2 g-2$ for $2 \leq g \leq 8$. Let $H$ be an induced subgraph of $B H_{n}$ with $|V(H)|=g+1$ and $|E(H)|=e_{g}\left(B H_{n}\right)$. By Lemma 2.2, suppose the bipartite graph $H=(X, Y)$, where $X$ and $Y$ are two parts of $H$. We divide our discussion into five cases.

Case 1. $2 \leq g \leq 4$

In this case, $|X|+|Y|=|V(H)|=g+1$. Hence,

$$
|E(H)| \leq|X| \cdot|Y| \leq\left\lfloor\frac{g+1}{2}\right\rfloor \cdot\left\lceil\frac{g+1}{2}\right\rceil=2 g-2 .
$$

Case 2. $g=5$.

In this case, $|X|+|Y|=|V(H)|=6$. Hence, $|E(H)| \leq|X| \cdot|Y| \leq 3 \times 3=9$. If $|E(H)|=9$, then $H$ is isomorphic to $K_{3,3}$, which contradicts Lemma 2.4. Thus, $|E(H)| \leq 8=2 g-2$.

Case 3. $g=6$.

In this case, $|X|+|Y|=|V(H)|=7$. Hence, $|E(H)| \leq|X| \cdot|Y| \leq 3 \times 4=12$. If $|E(H)|=12$, then $H$ is isomorphic to $K_{3,4}$, which contradicts Lemma 2.4. If $|E(H)|=$ 11, then $H$ is isomorphic to $K_{3,4}-e$ for some $e \in E\left(K_{3,4}\right)$, which also contradicts Lemma 2.4. Thus, $|E(H)| \leq 10=2 g-2$.

Case 4. $g=7$.

In this case, $|X|+|Y|=|V(H)|=8$. Hence, $|E(H)| \leq|X| \cdot|Y| \leq 4 \times 4=16$. Note that $\left|E\left(K_{2,6}\right)\right|=12=2 g-2$. Let $E_{0} \subseteq E\left(K_{3,5}\right)$ and $E_{1} \subseteq E\left(K_{4,4}\right)$. If $H=K_{3,5}-E_{0}$ and $\left|E_{0}\right| \leq 2$, then $H$ contains a subgraph isomorphic to $K_{3,3}$, which contradicts Lemma 2.4. If $H=K_{4,4}-E_{1}$ and $\left|E_{1}\right| \leq 3$, then by Lemma 2.5, suppose that $X=\left\{u_{1}, u_{1}^{\prime}, u_{2}, u_{2}^{\prime}\right\}$ and $Y=\left\{v_{1}, v_{1}^{\prime}, v_{2}, v_{2}^{\prime}\right\}$. Since $H=(X, Y)$ is an induced subgraph of $B H_{n},\left|E_{1}\right|=0$. Then $H$ is isomorphic to $K_{4,4}$, which contradicts Lemma 2.4. Thus, $|E(H)| \leq 12=2 g-2$.

Case 5. $g=8$.

In this case, $|X|+|Y|=|V(H)|=9$. Hence, $|E(H)| \leq|X| \cdot|Y| \leq 4 \times 5=20$. Note that $\left|E\left(K_{2,7}\right)\right|=14=2 g-2$. Let $E_{0} \subseteq E\left(K_{3,6}\right)$ and $E_{1} \subseteq E\left(K_{4,5}\right)$. Now, we only need 
to discuss the following two cases.

Case 5.1. $H=K_{3,6}-E_{0}$ and $\left|E_{0}\right| \leq 3$.

Note that $K_{3,6}-E_{0}$ with $\left|E_{0}\right| \leq 3$ contains a subgraph isomorphic to $K_{3,3}$, which contradicts Lemma 2.4 .

Case 5.2. $H=K_{4,5}-E_{1}$ and $\left|E_{1}\right| \leq 5$.

By Lemma 2.5, without loss of generality, suppose that $X=\left\{u_{1}, u_{1}^{\prime}, u_{2}, u_{2}^{\prime}\right\}$ and $Y=\left\{v_{1}, v_{1}^{\prime}, v_{2}, v_{2}^{\prime}, v\right\}$. Note that $H=(X, Y)$ is an induced subgraph of $B H_{n}$. Then $\left|E_{1}\right|$ can not be an odd integer. If $\left|E_{1}\right| \in\{0,2\}$, then $K_{4,5}-E_{1}$ contains a subgraph isomorphic to $K_{3,3}$, which contradicts Lemmas 2.4. If $\left|E_{1}\right|=4$ and $u_{i} v_{j} \in E_{1}$ for some $i, j \in\{1,2\}$, then edges $u_{i}^{\prime} v_{j}, u_{i} v_{j}^{\prime}, u_{i}^{\prime} v_{j}^{\prime} \in E_{1}$. Thus, $E_{1}=\left\{u_{i} v_{j}, u_{i}^{\prime} v_{j}, u_{i} v_{j}^{\prime}, u_{i}^{\prime} v_{j}^{\prime}\right\}$ and $H=K_{4,5}-E_{1}$ contains a subgraph isomorphic to $K_{3,3}$, which contradicts Lemmas 2.4. If $\left|E_{1}\right|=4$ and $u_{i} v_{j} \notin E_{1}$ for all $i, j \in\{1,2\}$, then $E_{1}=\left\{u_{1} v, u_{2} v, u_{1}^{\prime} v, u_{2}^{\prime} v\right\}$. Therefore, $H=K_{4,5}-E_{1}$ contains a subgraph isomorphic to $K_{3,3}$, which contradicts Lemmas 2.4.

Thus, $|E(H)| \leq 14=2 g-2$.

So, $e_{g}\left(B H_{n}\right)=2 g-2$ for $2 \leq g \leq 8$.

The following lemma gives a lower bound of $e_{g}\left(B H_{n}\right)$ for $9 \leq g \leq 2 n-1$, which will be used to disprove Conjecture 1.1 for $9 \leq g \leq 2 n-1$.

Lemma 3.5 The balanced hypercube $B H_{n}$ satisfies that $e_{g}\left(B H_{n}\right)>2 g-2$ for $9 \leq g \leq$ $2 n-1$.

Proof. To prove this lemma, it suffices to construct a subgraph of $B H_{n}$ with $g+1$ vertices and at least $2 g-1$ edges.

By Lemma 2.7, the girth of $X_{n}$ is 6 for $n \geq 3$. Suppose that $\overline{C_{6}}$ is a cycle of $X_{n}$ with six vertices. Let $H_{0}=\overline{C_{6}} \circ 2 K_{1}$ be a subgraph of $B H_{n}$. Since $B H_{n}$ is connected, by Lemma 2.6, $X_{n}$ is a connected graph for $n \geq 1$. Let $\overline{U_{t}}$ be a connected subgraph of $X_{n}$ with $\left|V\left(\overline{U_{t}}\right)\right|=t \geq 6$ satisfying that $\overline{U_{t}}$ is a unicyclic graph which contains $\overline{C_{6}}$. Then 
$\left|E\left(\overline{U_{t}}\right)\right|=t \geq 6$. Now, we distinguish the following four cases.

Case 1. $g=9$.

We consider the graph $H_{0}-\{u, v\}$, where $u, v \in V\left(H_{0}\right), u^{\prime} \neq v$ and $u v \in E\left(H_{0}\right)$. Note that $\left|V\left(H_{0}-\{u, v\}\right)\right|=g+1$ and $\left|E\left(H_{0}-\{u, v\}\right)\right|=2 g-1$. Then $e_{g}\left(B H_{n}\right) \geq$ $\left|E\left(H_{0}-\{u, v\}\right)\right|>2 g-2$.

Case 2. $g=10$.

We consider the graph $H_{0}-v$, where $v \in V\left(H_{0}\right)$. Note that $\left|V\left(H_{0}-v\right)\right|=g+1$ and $\left|E\left(H_{0}-v\right)\right|=2 g$. Then $e_{g}\left(B H_{n}\right) \geq\left|E\left(H_{0}-v\right)\right|>2 g-2$.

Case 3. $g$ is an odd integer with $g \geq 11$.

Since $g$ is an odd integer with $g \geq 11$, we have $\frac{g+1}{2} \geq 6$. We consider the graph $H_{g}=\overline{U_{\frac{g+1}{2}}} \circ 2 K_{1}$ as a subgraph of $B H_{n}$. Note that $\left|V\left(H_{g}\right)\right|=g+1$ and $\left|E\left(H_{g}\right)\right|=2 g+2$. Then $e_{g}\left(B H_{n}\right) \geq\left|E\left(H_{g}\right)\right|>2 g-2$.

Case 4. $g$ is an even integer with $g \geq 12$.

Since $g$ is an even integer with $g \geq 12, g-1$ is an odd integer with $g-1 \geq 11$. We consider the graph $H_{g-1}=\overline{U_{\frac{g}{2}}} \circ 2 K_{1}$ as a subgraph of $B H_{n}$. Pick a vertex $u$ from $V\left(B H_{n}\right) \backslash V\left(H_{g-1}\right)$. Note that $\left|V\left(H_{g-1}\right) \cup\{u\}\right|=g+1$ and $\left|E\left(B H_{n}\left[V\left(H_{g-1}\right) \cup\{u\}\right]\right)\right| \geq$ $\left|E\left(H_{g-1}\right)\right|=2 g$. Then $e_{g}\left(B H_{n}\right) \geq\left|E\left(B H_{n}\left[V\left(H_{g-1}\right) \cup\{u\}\right]\right)\right|>2 g-2$.

As mentioned above, we obtain the desired result.

Now, we give the proof of our main theorem.

Theorem 3.6 The g-extra edge-connectivity of balanced hypercubes $B H_{n}$ is $\lambda_{g}\left(B H_{n}\right)=$ $2(g+1) n-4 g+4$ for $n \geq 6-\frac{12}{g+1}$ and $2 \leq g \leq 8$. In addition, $\lambda_{g}\left(B H_{n}\right)<$ $2(g+1) n-4 g+4$ for $n \geq \frac{3 e_{g}\left(B H_{n}\right)}{g+1}$ and $9 \leq g \leq 2 n-1$.

Proof. By Lemma 2.8, $B H_{n}$ is edge-transitive. Note that $\left|V\left(B H_{n}\right)\right|=2^{2 n} \geq 6 n \geq$ $3(g+1)$ for $n \geq 2$. By Lemma 3.1, $\lambda_{g}\left(B H_{n}\right)=\gamma_{g}\left(B H_{n}\right)$ for $2 \leq g \leq 2 n-1$. 
By Lemma 3.4, $e_{g}\left(B H_{n}\right)=2 g-2$ for $2 \leq g \leq 8$. Since $n \geq 6-\frac{12}{g+1}$, we have $2 n \geq \frac{6(2 g-2)}{g+1}=\frac{6 e_{g}\left(B H_{n}\right)}{g+1}$ for $2 \leq g \leq 8$. By Lemma 3.2, $B H_{n}$ is $\gamma_{g}$-optimal. Thus, $\gamma_{g}\left(B H_{n}\right)=\beta_{g}\left(B H_{n}\right)$ for $n \geq 6-\frac{12}{g+1}$ and $2 \leq g \leq 8$. Since $\beta_{g}\left(B H_{n}\right)=2 n(g+1)-$ $2 e_{g}\left(B H_{n}\right)=2 n(g+1)-2(2 g-2)=2(g+1) n-4 g+4$, we have $\lambda_{g}\left(B H_{n}\right)=2(g+1) n-4 g+4$ for $n \geq 6-\frac{12}{g+1}$ and $2 \leq g \leq 8$.

By Lemma 3.5, $e_{g}\left(B H_{n}\right)>2 g-2$ for $9 \leq g \leq 2 n-1$. By Lemma 3.2, we have $\gamma_{g}\left(B H_{n}\right)=\beta_{g}\left(B H_{n}\right)=2 n(g+1)-2 e_{g}\left(B H_{n}\right)<2 n(g+1)-2(2 g-2)=2(g+1) n-4 g+4$ for $2 n \geq \frac{6 e_{g}\left(B H_{n}\right)}{g+1}$ and $9 \leq g \leq 2 n-1$. Therefore, $\lambda_{g}\left(B H_{n}\right)<2(g+1) n-4 g+4$ for $n \geq \frac{3 e_{g}\left(B H_{n}\right)}{g+1}$ and $9 \leq g \leq 2 n-1$.

\section{Conclusions}

The $g$-extra edge-connectivity is an important measure for the reliability of interconnection networks. We establish the $g$-extra edge-connectivity of balanced hypercubes $B H_{n}$, that is $\lambda_{g}\left(B H_{n}\right)=2(g+1) n-4 g+4$ for $n \geq 6-\frac{12}{g+1}$ and $2 \leq g \leq 8$, which partially confirms Conjecture 1.1. This result can provide a more accurate measurement of edge fault tolerance of balanced hypercubes. Meanwhile, we prove that $\lambda_{g}\left(B H_{n}\right)<2(g+1) n-4 g+4$ for $n \geq \frac{3 e_{g}\left(B H_{n}\right)}{g+1}$ and $9 \leq g \leq 2 n-1$, which disproves Conjecture 1.1 for any $n$ and $g$ with $n \geq \frac{3 e_{g}\left(B H_{n}\right)}{g+1}$ and $9 \leq g \leq 2 n-1$.

\section{Acknowledgement}

Y. Wei's research is supported by the Natural Science Foundation of Shanxi Province (No. 201901D211106). W. Yang's research is supported by the National Natural Science Foundation of China (No. 11671296). 


\section{References}

[1] J.A. Bondy, U.S.R. Murty, Graph Theory with Applications, The Macmillan Press Ltd, New York, 1976.

[2] A.H. Esfahanian, S.L. Hakimi, On computing a conditional edge-connectivity of a graph, Inform. Process. Lett., 27 (4) (1988), 195-199.

[3] J. Fàbrega, M.A. Foil, On the extraconnectivity of graphs, Discrete Math., 155 (1996), 49-57.

[4] Y.O. Hamidoune, A.S. Lladó, O. Serra, R. Tindell, On isoperimetric connectivity in vertex-transitive graphs, SIAM J. Discrete Math., 13 (2000), 139-144.

[5] P. Li, M. Xu, Fault-tolerant strong Menger (edge) connectivity and 3-extra edgeconnectivity of balanced hypercubes, Theoret. Comput. Sci., 707 (2018), 56-68.

[6] H.Z. Lü, On extra connectivity and extra edge-connectivity of balanced hypercubes, Int. J. Comput. Math., 94 (2017), 813-820.

[7] L.P. Montejano, I. Sau, On the complexity of computing the $k$-restricted edgeconnectivity of a graph, Theoret. Comput. Sci., 662 (2017), 31-39.

[8] M. Wang, Q. Li, On equivalence of isoperimetric edge connectivity and extra edge connectivity of graphs, J. Shanghai Jiaotong Univ., 36 (2002), 858-860.

[9] J. Wu, K. Huang, The balanced hypercube: a cube-based system for fault-tolerant applications, IEEE Trans. Comput., 46 (4) (1997), 484-490.

[10] J.M. Xu, Toplogical Structure and Analysis of Interconnection Networks, Kluwer Academic Publishes, Dordrecht/Boston/London, 2001.

[11] J.M. Xu, Combinatorial Theory in Networks, Science Press, Beijing/China, 2013. 
[12] D.W. Yang, Y.Q. Feng, J. Lee, J.X. Zhou, On extra connectivity and extra edgeconnectivity of balanced hypercubes, Appl. Math. Comput., 320 (2018), 464-473.

[13] M.C. Yang, Super connectivity of balanced hypercubes, Appl. Math. Comput., 219 (2012), 970-975.

[14] W. Yang, H. Lin, Reliability evaluation of BC networks in terms of the extra vertexand edge-connectivity, IEEE Trans. Comput., 63 (10) (2014), 2540-2548.

[15] M. Zhang, L. Zhang, X. Feng, H.J. Lai, An $O\left(\log _{2}(N)\right)$ algorithm for reliability evaluation of $h$-extra edge-connectivity of folded hypercubes, IEEE Trans. Reliab., 67 (1) (2018), 297-307.

[16] Z. Zhang, Extra edge connectivity and isoperimetric edge connectivity, Discrete Math., 308 (20) (2008), 4560-4569.

[17] J.X. Zhou, J. Kwak, Y.Q. Feng, Z.L. Wu, Automorphism group of the balanced hypercube, Ars Math. Contemp., 12 (1) (2017), 145-154.

[18] J.X. Zhou, Z.L. Wu, S.C. Yang, K.W. Yuan, Symmetric property and reliability of balanced hypercube, IEEE Trans. Comput., 64 (2015), 876-881.

[19] Q. Zhu, J.M. Xu, X.M. Hou, M. Xu, On reliability of the folded hypercubes, Inform. Sci., 177 (8) (2007), 1782-1788. 